\title{
Design of Miniaturized Sensors for a Mission-Oriented UAV Application: A New Pathway for Early Warning
}

\author{
Daniele Di Giovanni ${ }^{1,2 *}$, Francesca Fumian ${ }^{1,3}$, Andrea Chierici ${ }^{1,5}$, Mattia Bianchelli ${ }^{1,3}$, Luca Martellucci ${ }^{1}$, Gaetano \\ Carminati $^{1}$, Andrea Malizia ${ }^{4}$, Francesco d'Errico ${ }^{5,6}$, Pasqualino Gaudio ${ }^{1}$ \\ ${ }^{1}$ Department of Industrial Engineering, University of Rome Tor Vergata, via del Politecnico 1, Rome 00133, Italy \\ ${ }^{2}$ Unicamillus-Saint Camillus International University of Health Sciences, via di S. Alessandro 8, Rome 00131, Italy \\ ${ }^{3}$ Joint NBC Defence School, Piazza Marconi 7, 02100, Rieti, Italy \\ ${ }^{4}$ Department of Biomedicine and Prevention, University of Rome Tor Vergata, via Montpellier 1, Rome 00133, Italy \\ ${ }^{5}$ Department of Industrial and Civil Engineering, University of Pisa, Pisa 56122, Italy \\ ${ }^{6}$ Yale Center for Emergency Preparedness and Disaster Response, New Haven CT 06511, USA
}

Corresponding Author Email: daniele.di.giovanni@uniroma2.it

https://doi.org/10.18280/ijsse.110417

Received: 10 January 2021

Accepted: 12 July 2021

\section{Keywords:}

CBRN safety, chemical agents' detection, CRN agents sensing, unmanned aerial vehicles (UAV), SIBCRA mission

\begin{abstract}
In recent decades, the increasing threats associated with Chemical and Radiological (CR) agents prompted the development of new tools to detect and collect samples without putting in danger first responders inside contaminated areas. A particularly promising branch of these technological developments relates to the integration of different detectors and sampling systems with Unmanned Aerial Vehicles (UAV). The adoption of this equipment may bring significant benefits for both military and civilian implementations. For instance, instrumented UAVs could be used in support of specialist military teams such as Sampling and Identification of Biological, Chemical and Radiological Agents (SIBCRA) team, tasked to perform sampling in contaminated areas, detecting the presence of $\mathrm{CR}$ substances in field and then confirming, collecting and evaluating the effective threats. Furthermore, instrumented UAVs may find dual-use application in the civil world in support of emergency teams during industrial accidents and in the monitoring activities of critical infrastructures. Small size drones equipped with different instruments for detection and collection of samples may enable, indeed, several applications, becoming a tool versatile and easy to use in different fields, and even featuring equipment normally utilized in manual operation. The authors hereby present the design of miniaturized sensors for a mission-oriented UAV application and the preliminary results from an experimental campaign performed in 2020 .
\end{abstract}

\section{INTRODUCTION}

In recent decades, several events involving the release of chemical and radiological substances have occurred, such as accidental industrial losses in plants involving toxic chemical and mis-operation in nuclear plants or intentional release of chemical substances by non-state actors. Those accidents resulted in consequences for the personnel involved, including civilian and military first responders, such as fire brigades and defence specialist staff. In terms of the equipment provided to these operators, great progress has been made in portable detection and identification tools, now able to exploit several different operating principles and technologies. Nevertheless, especially during an early survey performed just after a chemical and radiological release, operators of national, international and intergovernmental organizations may be in a contaminated area without knowing the type and intensity of contamination, running the risk of facing still ongoing releases during the reconnaissance (recce) phase.

During these preliminary survey phases, another crucial aspect is timing: upon the notification of a release, the intervention team is activated, from this kick-off time several pre-deployment activities have to be completed and a security access authorization has to be received to avoid unacceptable risks for the team, including that of Improvised Explosive Devices (IED) to be cleared. In the case of OPCW inspection teams, several days or even weeks may pass before the necessary clearance is received. During this time, a loss of evidence regarding the dynamics of the incident may occur, especially if non-persistent chemical substances were involved.

On the other hand, the past few years have also seen the diffusion on a planetary scale of remotely piloted UAVs, with countless uses in different fields.

Drones may overcome the limitations mentioned above. Sending one or more GPS-fitted drones, classified as mini (Maximum take-off weight (MTOW) $<25 \mathrm{~kg}$ ), equipped with sensors able to detect and identify chemical compounds and radiological substances in real time, it is possible to geolocalize the point of detection by mean of devices to determine and communicate the exact position of the hotspot. Once the incident category is confirmed, environmental samples will be collected in a following mission identifying the compounds involved and the level of contamination by means of laboratory analysis. This approach may minimize or even completely avoid the exposure of personnel to contamination. Then, if a manned mission in field is still required, specialists 
will be provided with personal protective equipment chosen on the basis of the information collected by the UAV. Finally, waiting for the safety permissions for area entrance, time will be saved by an early UAV patrol.

The aerial platform may be equipped with a variety of sensors, capable of investigating and discriminating the nature of the threat for several classes of chemical and radiological contaminants. Some groups have reported the use of gas sensors mounted on a drone [1]. Different approaches have been compared for gas release localization with mobile platforms, and gas sensing systems have been developed and tested specifically for Volatile Organic Chemicals (VOC) [2] and Chemical Warfare Agents (CWAs) [3] localization and mapping. Numerous other applications have demonstrated the drones' suitability to be equipped with gas sensors for environmental monitoring and surveillance operations $[4,5]$, [6-11] and also for radiation monitoring after a nuclear disaster [12-14]. Hence, this study aimed at evaluating a drone sensor platform to increase first responders' safety during early survey missions and improve monitoring activities in critical contexts. In a later stage, our drone will be equipped with a sampling system capable of collecting evidence from different environmental matrices.

The paper is structured as follows: in Section 1 a brief overview about UAVs is given. Section 2 focuses on the interests of national, international and supra-national civil and military entities in the applications of this study. In Section 3 two different possible applications are investigated in conventional and non-conventional risk scenarios, such as industrial incidents and Chemical Biological Radiological Nuclear (CBRN) events. In Section 4, a case study is presented of in-field application of an UAV integrated with missionoriented payload tailored to the specific task of the mission. Firstly, the capabilities are described that a drone must provide to perform an initial survey of an area involved in a Chemical, Radiological and Nuclear (CRN) release. These may include miniaturized sensors for chemicals, Metal Oxide Detectors (MOX) and Photo-Ionization Detectors (PID) and radiological, as Scintillation Detectors. Secondly, the specific payload is explored which is required to perform a Sampling and Identification of Chemical and Radiological Agents (SICRA) mission in support of the SIBCRA team operation. This may include a miniaturized sampling system and identification technology such as Ion Mobility Spectrometry (IMS). Section 4 describes the preliminary results obtained, and Section 5 is dedicated to conclusions and future developments, defining the path for the integration of the whole system.

\subsection{UAVs}

UAVs have undergone significant technological developments in recent decades thanks to the advancements in research, technology and related applications. A wide range of flying machines are commercially available nowadays. Each different configuration meets several requirements, such as manoeuvrability, agility, max payload and operational time $[15,16]$. While UAVs have already been deployed successfully in military applications, their civil use has been receiving increasing attention by the robotics research community in the last few years [17-20]. Although UAVs already represent one of the most flexible solutions in a broad range of procedures, extensive technical and scientific research is needed to evaluate capability and limitations of these devices, for example in the choice of propeller design, available payload, operational range and power requirements.

A broad classification of drones is based on their wing type [21]. Between fixed and rotary wing UAV, the appropriate configuration must be chosen according to the nature, objective, and goals of the activity to be performed [22-24]. "Fixed wing" class has low power requirements, drones belonging to this class can fly at a high altitude and usually can carry high payload. However, this class of drones can only move forward, and this characteristic may not grant the required proximity to operate with specific sensors and requires a good knowledge of aerodynamics for piloting. Generally, these drones are relatively expensive. "Single Rotor wing" class drones are shaped like helicopters, are robust, and, compared to fixed wings, are able, with some piloting skills, to hover and change direction easily and may carry a significant payload. However, these are more energy-intensive, their wide rotor can be dangerous, and the turbulence they generate can be a limitation for certain types of sensors. The most widespread and commercially available category at present is the "Multi Rotor-wing" class, which is available in different configurations, sizes and payload options. A classification can be done according to the number of motors [21], the higher the number the better the stability. Quadcopter is the most common configuration [23], but hexa-copter and octa-copter are also frequently adopted. In terms of MTOW, drones of this class can carry high payloads and are also easy to manoeuvre. Moreover, take-off and landing are possible practically anywhere. Complex control systems, integrated with required hardware such as Inertia Measurement Unit (IMU), gyroscope and GPS, guarantee great stability. Thus, these drones offer the required features for the use of chemical detectors and air sampling systems [2527].

Furthermore, advancements in miniaturization of sensing devices, microprocessors as well as in actuator efficiency, have led to deployable aerial integrated systems that are appealing in term of usability, capability and cost. In addition, the development of autonomous piloting capabilities across a predetermined path through focal points, opens the way to a fully autonomous system, application for which UAVs are clearly better suited than manned aerial vehicle [25, 26, 28].

\section{NATIONAL, INTERNATIONAL AND SUPRA- NATIONAL CIVIL AND MILITARY ENTITIES INTEREST}

\subsection{Cooperation between university and military civil public entities}

The Quantum Electronics and Plasma Physics (QEPM) Research Group, within the Engineering Department of the University of Rome Tor Vergata, has been active since the early 60's in a variety of areas including: nuclear fusion, laser systems for indoor and outdoor monitoring and research activities on environment, energy and safety and security. The study presented in this paper stems from the expertise of the research group in the field of safety and security with a specific focus dedicated to $\mathrm{CBRN}$ risks.

In the field of CBRN, the QEPM research group [29] is a promoter of both research and education, and of training projects. In fact, for more than ten years it has been offering two postgraduate courses: International Master Level I and II 
in "Protection against CBRN events"; conducted, since 2009, by the group through the Department of Industrial Engineering and in collaboration with the School of Medicine and Surgery [30] of the same Academy.

The QEPM research group continuously establishes close links with national and international actors in the CBRN field (public and private Entities), supporting the creation of working groups connected to both its missions: technical research and scientific knowledge dissemination. In this framework, it promotes and runs bilateral and multilateral cooperation programs.

Among the collaborations established over the years by the research group, two are of particular relevance in the design of a drone for the CR detection and sampling. One is with The Italian Joint NBC Defence School located in Rieti. The other is with the Nuclear, Biological and Chemical NonProliferation of the Directorate General for Political and Security Affairs (DGAP) of the Ministry of Foreign Affairs and International Cooperation (MAECI), through its Office $V$ dedicated to Disarmament and Arms Control, which also includes the Italian National Authority (NA) for the Prohibition of Chemical Weapons (CWs).

Specifically, the long-term collaboration with the Italian Joint NBC Defence school started within the International Master CBRN for the development of teaching and training activities, and has been characterized by a strong synergy in major European projects in the field of CBRN (eNOTICE [31], RESIST [32], TRANSTUN [33]), as well as the sharing of $\mathrm{PhD}$ students and the development of research projects such as the drone for CR detection and sampling.

Similarly, the research group has begun to cooperate with MAECI DGAP Office V, and with its Italian NA for the Prohibition of CWs, in the context of the CBRN Master Courses. This collaboration has capped by successes such as the celebration of the $20^{\text {th }}$ anniversary of the Chemical Weapon Convention (CWC) during the Scientific International CBRN Conference (SICC) organized in 2017 [34] by the research group and, in the same year, with the honour of the international OPCW prize, the "The Hague Award" [35].

\subsection{OPCW "Research projects support programme" funding}

The development and testing activities of the UAV sensor platform for chemical detection and sampling has been funded within the framework of the OPCW "Research projects support program".

This program aims at supporting small-scale research for peaceful uses of chemistry in Member States. The OPCW Technical Secretariat grants funding to a limited number of projects either directly or jointly with co-funding institutions [36].

The main purpose of this program consists in the development and diffusion of Member States' scientific and technical competences in the field of chemistry for industrial, agricultural, research, medical, pharmaceutical, applications or other peaceful purposes not prohibited by the CWC.

The program's funds are dedicated to research in the areas of direct relevance to the implementation of the CWC and to those linked to Member States' priorities and sustainable development goals.

The development of this project aimed at increasing first responder's safety during early survey missions and improving monitoring activities in critical contexts. Moreover, the project may aid the inspection activities carried out within the CWC by OPCW inspectors.

\subsection{NATO "Defence Against Terrorism Programme of Work" funding}

Since its start, the project has been thought for dual-use applications. Indeed, even though detection and sampling applications appear interchangeable in the civil and military context, at an operational level, the scenarios of usage can be very different.

With this premise, development and testing activities for military deployment of the UAV payload equipped sensor for detection ( $\mathrm{C}$ and $\mathrm{R}$ ) and sampling (C), are conceived and conducted with the direct collaboration of the Italian Joint NBC Defence School, that obtained for this activity a grant within the framework of the NATO Defence Against Terrorism Programme of Work (DAT PoW).

This program was endorsed at the 2004 NATO Istanbul Summit and it aims at strengthening the Alliance's contribution to the fight against terrorism by strengthening capacity building, supporting operations and promoting partnerships. The main aim of the NATO's DAT PoW consists in preventing non-conventional attacks, such as suicide attacks with IEDs, and reducing other challenges, such as attacks on critical infrastructure [37]. The program relies on joint funding - member countries pool resources within a NATO framework, funded projects can be led by one nation or body and supported by others. The DAT PoW projects are organised under three capability umbrellas:

- Incident management

- Force protection and survivability

- Network engagement.

The development of CBRN defence capabilities, through the prevention of weapons of mass destruction proliferation and the defence against chemical, biological, radiological and nuclear $(\mathrm{CBRN})$ threats, falls within the Force Protection umbrella.

Ideally, Force Protection (FP) starts by stopping terrorists from obtaining and using CBRN weapons. If this prevention phase fails, the Alliance is committed to protect its forces, territory and populations and to offer recovery efforts. DAT PoW enhances the NATO countries' overall capability to comply with these commitments through projects addressing the main FP pillars: Detection, Identification and Monitoring (DIM) of CBRN substances, CBRN knowledge management, physical protection, hazard management and CBRN medical countermeasures. DAT PoW also supports the organization of training exercises, including those ones conducted with live agents.

\section{APPLICATION IN CONVENTIONAL AND NON- CONVENTIONAL RISK SCENARIOS}

In recent years, there has been a steady development and deployment of countless new uses of UAVs in the civil and military world, for example disaster management, geographical mapping of inaccessible places, aerial photography and express shipments. Moreover, UAVs integrated with thermal sensors are now employed daily by first responders to gather information during search and rescue operations. 
To perform the task of C-R detection and threats monitoring, a flying platform must be adequately equipped with a series of components to detect, and possibly identify and quantify the contamination. Furthermore, it would be desirable include a sampling system, able to withdraw evidence within several matrices for different classes of substances, in order to corroborate the detectors' assessment of the substances released.

In addition, during the early stages of disaster management, timeliness is crucial in evaluating accidental or intentional releases into the environment. Indeed, once a release has been noticed, before clearance is received to access the hotspot, several preliminary activities must be immediately completed. Delaying these activities by days or even hours may lead to loss of details about the accident dynamics, especially in case of highly volatile chemicals.

\subsection{Environmental monitoring application}

The use of drones in the field of environmental monitoring, such as for the monitoring of wide areas, such as landfill and critical infrastructures, is a topic of great interest in different fields [2, 6, 28, 38-41].

Indeed, UAVs may provide a solution for areal monitoring avoiding direct contact of first responders with an unknown release by offering operators the possibility of a rapid early warning in the event of a mistaken industrial operation, or attempted destruction by combustion of illegal solid waste, or open-air dispersal of gaseous waste.

The drone multisensor platform developed by the QEPM research group, through the selection of an appropriate set of sensors, can be used for this specific task.

\subsection{Application in case of a CBRN event}

The possibility of incidents connected to Toxic Industrial Chemical (TIC) and Toxic Industrial Radiological (TIR) accidental release and of TIC and TIR deliberate release in improvised devices during asymmetric conflicts is currently of great concern. To manage this threat, R\&D has occurred of specific tools for TIC and TIR detection, identification and sampling. UAVs equipped with $\mathrm{C} / \mathrm{R}$ detectors and sampling capabilities could solve a wide range of problems connected to first responder's safety, such as reducing human exposure in contaminated environments. The approach has not yet been thoroughly investigated for Chemical Warfare Agents [4].

The main aim of this study is the development of an instrumented drone, equipped with a payload for in-field monitoring, sampling and identification of the emission source and plume in case of incident.

As will be detailed hereafter, it was envisioned to employ the proposed system to support two types of missions; Survey missions and SICRA missions.

To determine the platform choice, a set of specific system requirements for the desired goal of this work have been considered. The UAV must be able to perform a high proximity flight to the release point, to lower the distance from the target and have the possibility to perform the substance identification. In addition, several crucial indicators have to be taken into account, such as energy consumption, available payload, duration of flight compatible with the mission accomplishment. On this basis, the most suitable drone is a rotary wing. Numerous previous studies have shown that this configuration provides the right balance between the desired requirements $[1,2,5]$.

To prevent the need for a professional aircraft pilot, a UAV in the "mini-micro-UAV" category (MTOW: $25 \mathrm{~kg}$ ) was identified as the most suitable solution.

Concerning chemical detection, within the wide range of available working principles, two different technologies (PID and MOX) have been examined for this sub-system. By combining these two working principles, it is possible to acquire a qualitative identification of the class of substances in the release and to quantify the contamination level against a given threshold. Indeed, both working principles are characterized in terms of their reliability to assess concentrations of a known agent in the open air, high sensitivity, low weight, and low cost. Used in combination, they may offer complementary recognition capabilities.

Concerning radiological detection, within the wide range of possibilities offered by the available working principles, a custom-made or partially integrated solution based on inorganic crystal scintillators (such as $\mathrm{Cs}(\mathrm{Tl})$ ) has been selected as the gamma radiation detection technology of choice. While fully integrated solutions are available on the market, they are expensive and may be difficult to interface with other subsystems present in the platform. Inorganic scintillators, once coupled with a proper light detection device (PIN diodes, APDs, or SiPMs), provide a relatively high detection efficiency in a small active volume; moreover, by developing a data acquisition system able to discriminate the energy of the incoming gamma photons, it is possible to perform both a quantitative and qualitative (i.e., radiological or nuclear source identification) analysis of the radioactive contamination

Other instruments, whether for chemical or radiological detection, were not investigated because they were considered too heavy or bulky to be integrated on a mini-category drone. Some others were not considered because they are not compatible with use in flight, due to their operating principle that uses radiological sources as ionization unit. Others yet, are not applicable in the case of release of flammable or explosive substances, for which the detector may become an ignition point.

Regarding the best solution for UAV sensor positioning, studies were done with the aid of computational fluid-dynamic simulations. These models were used to evaluate the best compromise between drone flyability and rotors influence on detection and identification capabilities. A specific study on the best sensor's location for radioactive release detection has been published already [42]. A separate study is in progress assessing the best positioning of sensors for chemical detection dispersion.

\section{A CASE STUDY: UAV WITH INTEGRATED DETECTOR AND SAMPLING DEVICE FOR SURVEY AND SICRA MISSION}

\subsection{Survey mission}

An early substance detection would allow hotspot identification and release-plume tracking which would be invaluable in areas susceptible to contamination, such as industrial districts or locations with unstable governments and non-state actors possibly using non-conventional agents.

This Survey mission could be performed by a micro drone equipped with low-cost and low-weight PID and MOX sensors 
able to patrol a wide area and detect any deviation from an environmental baseline to give a first alarm, possibly substituting an in-field reconnaissance human team in the early survey phases.

Figure 1 provides a schematic representation of the overall system integration architecture for survey missions.

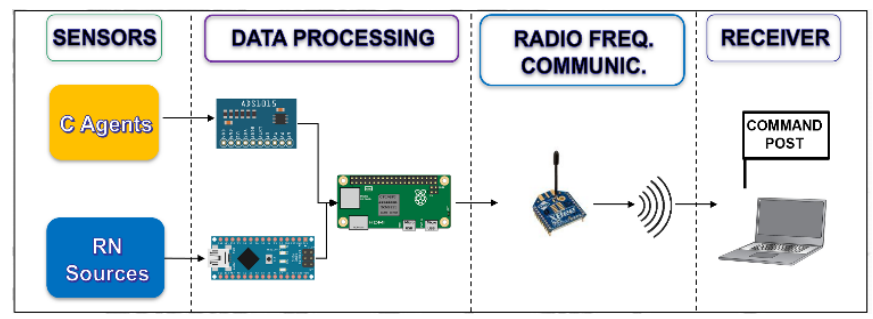

Figure 1. Schematic representation of the system architecture for survey operations

\subsubsection{Photo-Ionization Detectors (PID)}

The first operating principle chosen for this application is the PID. Detectors using PID are effective in detecting and monitoring many hazardous substances and are generally inexpensive. Furthermore, they offer a fast response [43]. Compared to several other hazardous gas detection methods available on the market, PIDs offer a combination of response rates, ease of use and maintenance, compact size and ability to detect low concentrations, including most VOCs. PIDs use the CAs ionization working principle. When the sample gas absorbs energy from a PID lamp, the gas is excited, and its molecular content is altered.

The compound drops an electron $\left(\mathrm{e}^{-}\right)$and changes into a positive ion [44]. After this process, the substance is ionized. Most substances can be ionized, some of them more easily than others.

To ionize the compound being monitored, PIDs use an ultraviolet (UV) lamp. The lamp, oftentimes the size of a common flashlight bulb, is designed to emit at a specific voltage for example, $10.6 \mathrm{eV}$ lamp emits enough UV energy to ionize any compound with an IP value below $10.6 \mathrm{eV}$ [45].

Measuring the current produced by the ionized compounds enables the assessment of concentrations as low as parts per million (ppm). PID detectors are capable of measuring most organic compounds and some inorganic compounds, including ammonia and sulfuric acid. These detectors are widely used in many different scenarios, such as leaks from industrial equipment, perimeter monitoring of industrial buildings, or warehouses if the nature of spill is known, delineation of contaminated areas after a release, technical investigations after a fire.

Thanks to their sensitivity and fast response, these instruments are highly useful both for first responder [46] and CWA applications [47].

PID detectors are capable of measuring the concentration of a known gas dispersed in the atmosphere, but they have no identification capability. In fact, they can be used to give meaningful quantitative information, but they must be coupled with an instrument capable of substance identification (qualitative assessment).

A very small number of applications have been conducted previously with the integration of these sensors with UAS, most of them dedicated to investigating combustion products $[6,48]$ or particulate matter [7]. Regarding industrial pollutants, the use has been reported of electrochemical sensors [8], which are less sensitive than PID to VOC.

\subsubsection{Metal-Oxide sensors (MOX)}

MOX sensors are based on variations in the conductivity of a semiconductor layer due to the oxidation of a gas [49-51]. This electrochemical gas sensor measures the concentration of target gas by an oxidization process at an electrode that produces a variation in the current flow. Therefore, the degree of oxidation of the target gas causes a change in the conductivity of the semi-conductor layer that can be measured through an external electronic acquisition device. The gas diffuses into the sensor through a porous membrane or through an inlet, and it reaches the working electrode where it is oxidized. This electrochemical reaction results in a current that passes through the circuit. The amount of current that flows between working electrodes is determined by how much of the target gas is oxidized.

The specific MOX gas-sensitive layer reacts to oxidizing gases with an increase of the layer resistance and reacts to reducing gases with a lowering of the layer resistance. According to the type of gas and the sensor type, a specific MOX gas sensor responds to concentrations from a few $\mathrm{ppb}$ up to ppm [52]. This kind of response together with the low sensor cost, offers a significant advantage over other sensors, i.e., infrared based, where the output must be processed before they can be used to measure concentrations. The most significant reason for choosing MOX gas sensors for a preliminary in-field contamination analysis, lies in the low cost and fast deployability of this kind of sensor.

\subsubsection{Gamma radiation detectors}

Inorganic scintillation crystals (such as $\mathrm{Cs}(\mathrm{Tl})$ ) are a favoured choice for gamma detection and spectroscopy since they provide high detection efficiency in a small active volume. In scintillation spectroscopy, the energy spectrum of a gamma ray source is measured by converting gamma rays into pulses of visible light in a scintillator material. Subsequently, the light pulses are transduced into electrical signals by a low-level light detector coupled to the scintillator. Common choices for the light detector include a Photo-Multiplier Tube (PMT), PIN photodiode, avalanche photodiode (APD), or more recently, silicon photomultipliers (SiPM) [53].

The light emission of the scintillator decays on a time scale that is a characteristic of the material used, ranging from tens of ns to several $\mu$ s. Commonly, the light pulse is converted into an electrical signal, and a Charge Sensitive Preamplifer (CSP) is used to integrate the current pulse produced by this light detector into a voltage pulse. The voltage pulse height of the CSP is proportional to the total charge released by the photoelectron. In this configuration, the output voltage of the CSP, namely the pulse height, is a measure of the ionization energy deposited in the scintillator by the gamma ray.

Different approaches can be used to measure and process the CSP output voltage: often, the signal-to-noise ratio of the acquisition chain is improved by using a gaussian Shaping Amplifier (SA) on the CSP signal before the pulse height is measured. The SA operates as a bandwidth filter and of a signal amplifier. Furthermore, due to the high-pass component of the filter, the long tail from the CSP output is eliminated, allowing the pulse-pileup effect between events close in time to be mitigated. Finally, to collect and digitize such signals the conversion technique of choice always involves a trade-off between the available hardware resources, costs, space and power consumption.

The main reason for the choice of this system to give a first alarm in case of suspected radiological release, lies in its low 
weight, low cost and reliable sensitivity.

\subsection{SICRA mission and its integration with SIBCRA team}

Once evidence of contamination is obtained through the Survey mission and an alarm is issued, a Sampling and Identification of $\mathrm{C} / \mathrm{R}$ agents (SICRA) mission can be initiated, proceeding to an event data collection with a due chain of custody. The SICRA mission may be performed by a minidrone equipped with specific mission-oriented payload, modular and flexible depending on the nature of the alarm received, able to identify with confidence the source of contamination. Moreover, with a customized sampling system representative evidence may be collected from different environmental matrixes for subsequent laboratory analysis.

The SIBCRA Team (Sampling and Identification of Biological, Chemical and Radiological Agents) comprises specialized CBRN personnel with a theoretical and practical knowledge about CBR sample collection and management procedures as foreseen by NATO doctrine AEP-66 (STANAG 4701) [54] and forensic science [55].

The main SIBCRA team tasks are as follows:

- carrying out the initial reconnaissance aimed at identifying and confirming hot spots in the contaminated area [56];

- collecting determined samples, consistent in matrix and quantity (e.g., water, liquids, soil, etc.) including control samples;

- complying with protocols envisaged for sample packaging and for their subsequent safe transportation and filling in the required forms to identify the samples (data sheet) and accompany their transport (chain of custody);

- delivering the samples directly to the reference laboratories (national/NATO/international) or to the transport personnel in the shortest possible time [57].

A SICRA UAV can integrate the activities of the SIBCRA Team by collecting evidence in scarcely accessible areas or dangerous sites, by reducing personnel exposure.

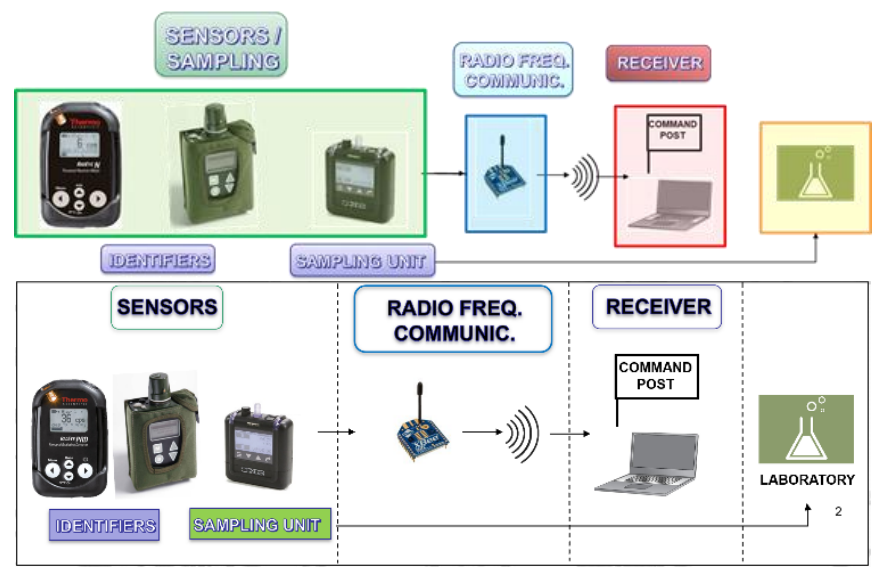

Figure 2. Schematic representation of the system architecture for SICRA operations.

The drone can take-off following a preliminary assessment done by the survey mission, which evaluates which kind of threat is present. The drone may also be deployed directly as a primary mission, if news of a chemical incident or of a radiological dispersion has been reported by individuals in the field or by a manned survey. In either case, the SICRA mission will be equipped with features specifically selected and designed for the threat preliminarily assessed, whether if it is $\mathrm{R}$ or $\mathrm{C}$.

The possible use of survey or SICRA drones would allow a time reduction for the monitoring activity, with a reduction or even complete avoidance of the exposure of personnel. This way, the Team, in compliance with the short mission time, can reduce the search time in order to dedicate himself to more accurate or specifical sampling activities.

Figure 2 presents the schematic representation of the integration architecture of the overall system for SICRA missions.

\subsubsection{Ion-Mobility Spectrometry (IMS)}

The working principle chosen for chemical detection in the SICRA mission is Ion-Mobility Spectrometry (IMS). This represents an established technology employed in several instruments used as portable detectors [58].

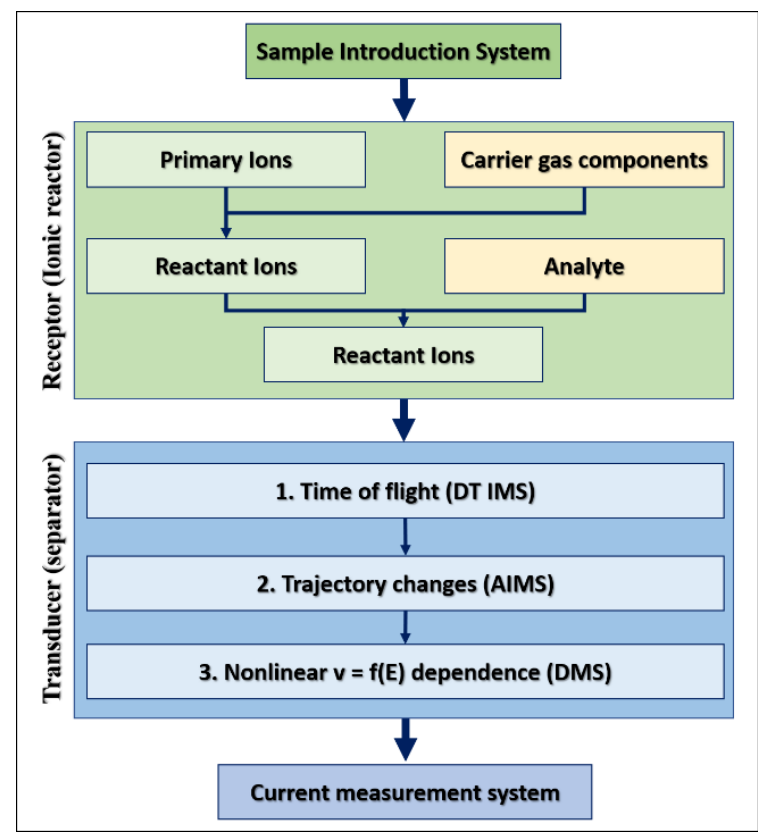

Figure 3. Ion mobility spectrometry (IMS) — operation principle/measurement systems elements

IMS analysers are devices designed to be selective thanks to the different effects of the ionization process on each chemical substance. Consequently, they enable the discrimination of different components in an air sample. The operating principle of the IMS involves two main stages. The first comprises collection of the sample and its ionization in an ion reactor, with the generation of ions containing analyte molecules or their fragments. The second step comprises the separation of the ions, which takes place in the transducer section of the detector (Figure 3). The ion separation step differentiates IMS from other simpler ionization techniques, as, i.e., Flame Ionization Detectors (FID) and Electron Capture Detectors (ECD) [58]. The flow of ions produced in the reactor into the collector generates the output signal. This ion movement takes place in a carrier gas flow (advection) and an electric field (drift)

For integration with an UAV, corona discharge [59] is recommended instead of radioactive sources as ionizing agents. The latest must be avoided, in view of the regulation on the transport of dangerous goods by air [60]. 
Among commercially available portable IMS detectors that do not adopt radioactive sources as an ionization method, the SMITH Detection LCD3.3 instrument was deemed a suitable solution. In a previous study, this technology was used to assess the diffusion of methyl salicylate in air [9]. The LCD3.3 was powered by an AA battery and performed continuous air sampling and analysis [61]. Considering its selectivity and sensitivity, the IMS, in conjunction with the quantitative response given by the PID, can provide a qualitative answer regarding the nature of the release.

\subsubsection{Gamma radiation identifier}

Regarding the case of radiological incident, the gamma radiation detector working principle under development described in section 4.1.3 is suitable to perform also a SICRA mission. Indeed, this miniaturized system is also able, thanks to its data-processing unit, to perform the identification of the detected isotopes.

Moreover, it may be certainly possible to employ commercially available handled solutions, such as the Radeye (Thermo Scientific) or the Identifinder R400 (Flir System). However, by choosing those instruments, a compromise in terms of higher costs and weight has to be accepted in comparison with Scintillator detector.

\subsubsection{Miniaturized sampling systems for UAV application}

To select an effective sampling system choice, several technical specifications already highlighted for the other subsystems as being lightweight, offering plug-and-play operation, low cost and low power consumption are still valid guidelines. There are many technological solutions, developed for environmental monitoring applications, to perform the collection of representative specimens, some of those may be suitable for CBRN applications. The primary objective is to get a sample back to a laboratory from the place marked as the origin of the release and recognized by the other instruments integrated in the UAV for the characterization and confirmation of the evidence. A multi-sampling system working in parallel is proposed consisting of a device that simultaneously collects unwanted CWAs or TICs and particulate matter, the system must preserve the characteristics of the sample until it is analysed. The system is expected to ensure the capability of selectively retaining different substances, e.g., sintered cartridge sampling systems [62], which are either disposable or reusable after desorption, giving the possibility to sample from different environmental matrices, gaseous matrix and aerosol samples. Available commercial solutions with this feature include air-sampling pumps. These devices are used as personal equipment for environmental monitoring and provide low flows from 20 to $500 \mathrm{~mL} / \mathrm{min}$ for sampling gases and vapours, some models offer also Bluetooth connectivity allowing remote sampling programming [63]. Air-sampler pumps are available with limited weight, generally less than $250 \mathrm{~g}$, which can operate with different sampling media, such as dust trap impinger or sintered metal cartridges capable of immobilizing VOC components and pollutants. The latter configuration allows the use a low flow tube holder to manage many samples in parallel and offers the possibility to replicate the analysis once the sample is withdrawn from the laboratory.

From the commercially available portable air-sampler pumps, the Pocket Pump Touch SKC was deemed suitable for our applications. This pump features an inlet flowrate from 20 to $500 \mathrm{ml} / \mathrm{minute}$ on coconut charcoal as adsorbent medium. This adsorbent bed is compatible both with the chemicals to be sampled that with the solvents used for chemical desorption.

As regards the integration of the sampling system into the UAS, it is possible to implement the same interface used for the PID detectors, an Arduino microcontroller, to control the duration, nature, and flow rate of the sampling in real-time.

\subsection{Preliminary experimental results}

Preliminary field tests were carried out to assess the behaviour of the LCD3.3 detector when integrated with a commercial rotary-wing UAV (DJI Matrice 210) (Figure 4a)). A nerve agent simulant [64] deployed in the open field was used to activate the instrument channel corresponding to volatile nerve agents (in NATO standard acronym GB) [65], the one devoted to organophosphates. This class of chemicals is commonly employed as insecticides in agriculture, but, if used in concentrations exceeding the Limit of Exposure (LoE) acceptable by law, they can seriously injure human health.

In these tests, a release scenario has been simulated. In the simulation, a command post received an alarm concerning the intentional release of chemical agents by means of a mortar hit with a special charge, as could be observed from the visible release plume. Based on the information received from the witnesses, a SICRA mission, equipped with a specific payload for chemical identification and sampling, was sent to the location recognized to be the hotspot.

First of all, a hovering of the area was performed to assess the situation and pinpoint the hot spot. After that, the pilot proceeded to a proximity flight, in order to approach the contamination and assess the entity and sample the release for a later analysis.

As shown in Figure 4b, the selected IMS instrument gave a good response in terms of detection when integrated into a UAS. This device is typically used for manned applications by first responders and specialists from armed forces. Indeed, with a strong signal for GB agent, the chemicals class was identified. Using the detector connected to a pc, a comparison of the peak altitude of the detected substance with the Reactant Ion Peak (RIP) also gave an approximate quantification of the concentration of the agent in air.
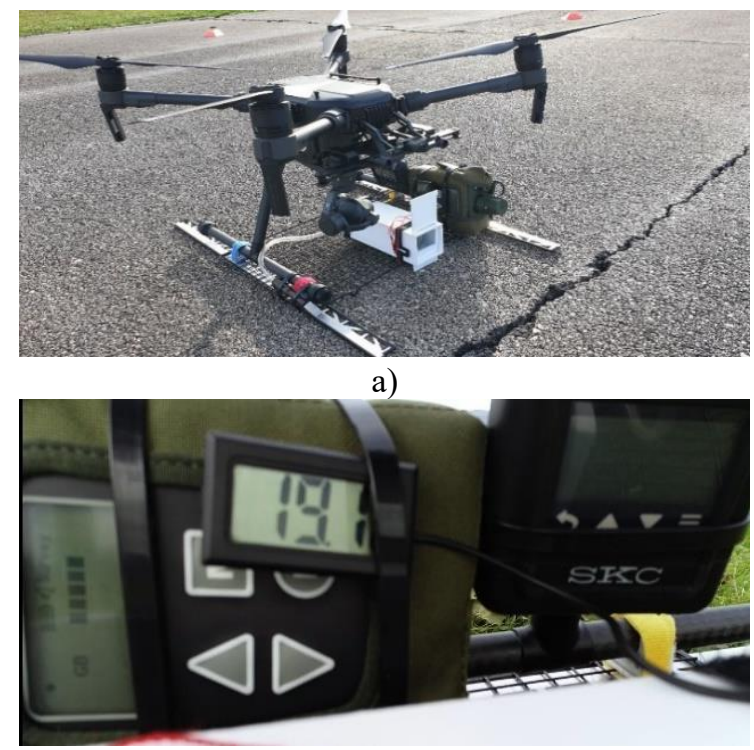

b)

Figure 4. (a) The field test campaign setup; (b) the display view of LCD3.3 detector on the alarm 


\section{CONCLUSIONS AND FUTURE DEVELOPMENTS}

In a historical moment characterized by high technological and industrial development, but also by great inequalities and a high number of social and economic conflicts, our communities are more exposed to the risk of malicious actions and accidents. The main objective of this research is to develop a flexible, low-cost platform that can be easily deployed in different CR scenarios. General layouts have been designed considering different potential applications: industrial contexts, critical infrastructures, landfills to be monitored, avoiding illegal spillage of hazardous substances, or the attempt of destruction by fire of unauthorized waste. The capability to interface and intercommunicate different subsystems, each of which has a specific function, extends the range of possible missions to be performed.

By going through the various subsystems presented, a properly instrumented UAV can be used for monitoring critical areas, where there is a suspicion that there may have been a release.

The detection techniques considered suitable for this integration, PID, MOX and IMS, allow a good balance between the different operational requirements indicated. In fact, their coupling allows to achieve, with high confidence, a quantitative (concentration with PID and MOX), and a qualitative (identification with IMS) measurement of the detected release.

In addition, the integration of a sampling system on the UAV makes it possible to collect representative samples for subsequent laboratory analysis and confirmation of the previous results obtained through field measurements.

The proposed multi-sensor platforms for UAVs can be organized according to the type of mission. For survey missions MOX and PID sensors Scintillation Detectors can be used, very light and cheap, able to provide alarms in case of detection of high concentrations of potentially dangerous substances, which can be loaded on small and inexpensive drones.

For SICRA missions (following the completion of a survey mission that resulted in an alert), a different set-up of the multisensor platform is envisaged, with the possibility of identifying the released substance (with IMS) and sampling, to carry out further analysis in the laboratory.

A confirmation of the validity of the different configuration hypotheses, will require extensive test campaigns. A first set of measurements has already been performed and was aimed at verifying the correct operation of the detection and identification features on board, providing some preliminary feedback.

Preliminary tests carried out on the instrumented flying platform proved that the chosen instrument integration hypothesis is suitable for this application and opens the path to possible new experiments. Therefore, the flying platform equipped with light and low-cost payload integrating sensors and sampling systems makes it possible to reduce the risks associated with deploying human operators in hazardous environments.

Furthermore, the opportunity to collect samples of contaminants allowed us to intercompare in-field measurements with laboratory analyses [66].

Several test campaigns are currently in progress to investigate the suitability of the integration of MOX and PID sensors and drones.

Other tests are conducted to investigate the sensors' position on the UAS in order to improve detection and identification capabilities. These experiments are designed to verify what was determined through previous numerical fluid dynamics simulations $[42,67]$ aimed at examining rotatory wings UAV turbulence in the presence of various hazardous chemicals.

\section{ACKNOWLEDGMENT}

This work was funded in part by the Organization for Prohibition of Chemical Weapons (OPCW) within the programme "Support for Research Programme" 2018 and by NATO with a grant to the NBC School of Rieti (Italian Ministry of Defence) managed by the University of Rome Tor Vergata in the 2019 "Defence Against Terrorism Programme of Work" (DAT PoW).

We would like to thank the Joint NBC Defence School of Rieti and its staff for the availability of their facilities in conducting of the experimental tests, the Quantum Electronics and Plasma Physics Research Group the technical support in the development activities and Heli Protection Europe s.r.l. for the technical support in the flight activities and for providing the flying platform.

\section{REFERENCES}

[1] Rosser, K., Pavey, K., FitzGerald, N., Fatiaki, A., Neumann, D., Carr, D., Chahl, J. (2015). Autonomous chemical vapour detection by micro UAV. Remote Sensing, $\quad$ 7(12): 16865-16882. https://doi.org/10.3390/rs71215858

[2] Rossi, M., Brunelli, D., Adami, A., Lorenzelli, L., Menna F., Remondino, F. (2014). Gas-drone: Portable gas sensing system on UAVs for gas leakage localization. Sensors, $\mathrm{pp}$. https://doi.org/10.1109/ICSENS.2014.6985282

[3] Rabajczyk, A., Zboina, J., Zielecka, M., Fellner, R. (2020). Monitoring of selected CBRN threats in the air in industrial areas with the use of unmanned aerial vehicles. Atmosphere, 11(12): 1373. https://doi.org/10.3390/atmos11121373

[4] Karpowicz, J. (2016). Detecting radiological, biological and chemical threats with drones. Commercial UAV News. Available:

https://www.commercialuavnews.com/publicsafety/detecting-radiological-biological-chemicalthreats-drones.

[5] Nikolic, J., Burri, M., Rehder, J., Leutenegger, S., Huerzeler, C., Siegwart, R. (2013). A UAV system for inspection of industrial facilities. In 2013 IEEE Aerospace Conference, 1-8. https://doi.org/10.1109/AERO.2013.6496959

[6] Bolla, G.M., Casagrande, M., Comazzetto, A., Dal Moro, R., Destro, M., Fantin, E., Lorenzini, E.C. (2018). ARIA: Air pollutants monitoring using UAVs. In 2018 5th IEEE International Workshop on Metrology for AeroSpace (MetroAeroSpace), pp. 225-229. https://doi.org/10.1109/MetroAeroSpace.2018.8453584

[7] Bezantakos, S., Schmidt-Ott, F., Biskos, G. (2018). Performance evaluation of the cost-effective and lightweight Alphasense optical particle counter for use onboard unmanned aerial vehicles. Aerosol Science and Technology, 52(4): 385-392. 
https://doi.org/10.1080/02786826.2017.1412394

[8] Gu, Q., R Michanowicz, D., Jia, C. (2018). Developing a modular unmanned aerial vehicle (UAV) platform for air pollution profiling. Sensors, 18(12): 4363. https://doi.org/10.3390/s18124363

[9] Marinelli, W.J., Schmit, T., Dupuis, J.R., Mulhall, P., Croteau, P., Manegold, D., Lav, M. (2015). Cooperative use of standoff and UAV sensors for CBRNE detection. In Chemical, Biological, Radiological, Nuclear, and Explosives (CBRNE) Sensing XVI, 9455: 94550U. https://doi.org/10.1117/12.2177023

[10] Fumian, F., Di Giovanni, D., Martellucci, L., Rossi, R., Gaudio, P. (2020). Application of miniaturized sensors to unmanned aerial systems, a new pathway for the survey of polluted areas: Preliminary results. Atmosphere, 11(5): 471. https://doi.org/10.3390/atmos11050471

[11] Do, S., Lee, M., Kim, J.S. (2020). The effect of a flow field on chemical detection performance of quadrotor drone. $\quad$ Sensors, $20(11)$ : 3262 https://doi.org/10.3390/s20113262

[12] Sanada, Y., Torii, T. (2015). Aerial radiation monitoring around the Fukushima Dai-ichi nuclear power plant using an unmanned helicopter. Journal of Environmental Radioactivity, 139: 294-299. https://doi.org/10.1016/j.jenvrad.2014.06.027

[13] Towler, J., Krawiec, B., Kochersberger, K. (2012). Radiation mapping in post-disaster environments using an autonomous helicopter. Remote Sensing, 4(7): 19952015. https://doi.org/10.3390/rs4071995

[14] Kurvinen, K., Smolander, P., Pöllänen, R., Kuukankorpi, S., Kettunen, M., Lyytinen, J. (2005). Design of a radiation surveillance unit for an unmanned aerial vehicle. Journal of Environmental Radioactivity, 81(1) 1-10. https://doi.org/10.1016/j.jenvrad.2004.10.009

[15] Feron, E., Johnson, E.N. (2008). Aerial Robotics. Springer, Springer Handbook of Robotics.

[16] International Conference on Modelling and Simulation for Autonomous Systems.

[17] Nonami, K., Kendoul, F., Suzuki, S., Wang, W., Nakazawa, D. (2010). Autonomous flying robots: Unmanned aerial vehicles and micro aerial vehicles. Springer Science \& Business Media.

[18] Valasek, J. (2013). Small Unmanned Aircraft: Theory and Practice. Princeton University Press.

[19] Siegwart, R., Nourbakhsh, I.R., Scaramuzza, D. (2011). Introduction to Autonomous Mobile Robots. MIT press.

[20] Siciliano, B. (2009). Mobile Robots. Robotics: Modelling, Planning and Control, 469-521.

[21] Fahlstrom, P., Gleason, T. (2012). Introduction to UAV Systems. John Wiley \& Sons.

[22] Gurdan, D., Stumpf, J., Achtelik, M., Doth, K.M., Hirzinger, G., Rus, D. (2007). Energy-efficient autonomous four-rotor flying robot controlled at $1 \mathrm{kHz}$. In Proceedings 2007 IEEE International Conference on Robotics and Automation, pp. 361-366. https://doi.org/10.1109/ROBOT.2007.363813

[23] Hoffmann, G., Huang, H., Waslander, S., Tomlin, C. (2007). Quadrotor helicopter flight dynamics and control: Theory and experiment. In AIAA Guidance, Navigation and Control Conference and Exhibit, 6461. https://doi.org/10.2514/6.2007-6461

[24] Tomic, T., Schmid, K., Lutz, P., Domel, A., Kassecker, M., Mair, E., Burschka, D. (2012). Toward a fully autonomous UAV: Research platform for indoor and outdoor urban search and rescue. IEEE Robotics \& Automation Magazine, 19(3): 46-56. https://doi.org/10.1109/MRA.2012.2206473

[25] Mettler, B. (2002). Identification, Modeling and Characteristics of Miniature Rotorcraft, Boston: Springer Science + Business Media, LLC.

[26] Achtelik, M., Doth, K.M., Gurdan, D., Stumpf, J. (2012). Design of a multi rotor MAV with regard to efficiency, dynamics and redundancy. In AIAA Guidance, Navigation, and Control Conference, 2012: 4779. https://doi.org/10.2514/6.2012-4779

[27] Doherty, P., Kvarnström, J., Heintz, F. (2009). A temporal logic-based planning and execution monitoring framework for unmanned aircraft systems. Autonomous Agents and Multi-Agent Systems, 19(3): 332-377. https://doi.org/10.1007/s10458-009-9079-8

[28] Bachrach, A., Prentice, S., He, R., Henry, P., Huang, A.S. Krainin, M., Roy, N. (2012). Estimation, planning, and mapping for autonomous flight using an RGB-D camera in GPS-denied environments. The International Journal of Robotics Research, 31(11): 1320-1343. https://doi.org/10.1177/0278364912455256

[29] QEPM. Quantum Electronics and Plasma Physics Research Group. Available: https://www.qepresearch.it/

[30] HESAR. Master CBRNe. Available: https://www.cbrngate.com/master-cbrne/

[31] European Network of CBRN TraIning CEnters. Available: https://www.h2020-enotice.eu/

[32] REsilience Support for critical Infrastructures through Standardised Training on CBRN. Available: https://project-resist.eu/

[33] TRANSnational TUNnel operational CBRN risk mitigation. Available: https://transtun-project.eu/

[34] S. 2. L. O. Committee. 1st Scientific International Conference on CBRNe. Available: https://www.siccseries.com/old-conference-sicc2017/.

[35] OPCW. Winners of 2017 OPCW-The Hague Award Announced. Available: https://www.opcw.org/mediacentre/news/2017/11/winners-2017-opcw-hague-awardannounced.

[36] OPCW. Capacity Building Research Project Support Programme. Available: https://www.opcw.org/resources/capacitybuilding/international-cooperationprogrammes/research-project-supportprogramme\#: :text=Supporting\%20small\%2Dscale $\% 20$ research $\% 20 \mathrm{in}$,jointly $\% 20$ with $\% 20 \mathrm{co} \% 2 \mathrm{Dfunding} \% 20 \mathrm{i}$ nstitutions

[37] NATO. Defence Against Terrorism Programme of Work (DAT POW). Available: https://www.nato.int/cps/en/natohq/topics_50313.htm\#: $\sim:$ text $=$ The $\% 20$ aim $\% 20$ of $\% 20$ the $\% 20$ Alliance's, as $\% 20$ attacks\%20on\%20critical\%20infrastructure

[38] Asad, M., Al Aidaros, O., Beg, R., Al Dhahri, M., Al Neyadi, S., Hussein, M. (2017). Development of autonomous drone for gas sensing application. In 2017 International Conference on Electrical and Computing Technologies and Applications (ICECTA), pp. 1-6. https://doi.org/10.1109/ICECTA.2017.8252068

[39] Burgués, J., Marco, S. (2020). Environmental chemical sensing using small drones: A review. Science of the Total Environment, 141172. https://doi.org/10.1016/j.scitotenv.2020.141172

[40] Baumbach, J.I., Sielemann, S., Xie, Z., Schmidt, H. 
(2003). Detection of the gasoline components methyl tert-butyl ether, benzene, toluene, and m-xylene using ion mobility spectrometers with a radioactive and UV ionization source. Analytical Chemistry, 75(6): 14831490. https://doi.org/10.1021/ac020342i

[41] Marconi, L., Naldi, R., Gentili, L. (2011). Modelling and control of a flying robot interacting with the environment. Automatica, 47(12): 2571-2583. https://doi.org/10.1016/j.automatica.2011.09.020

[42] Marturano, F., Ciparisse, J.F., Chierici, A., d'Errico, F., Di Giovanni, D., Fumian, F., Malizia, A. (2020). Enhancing Radiation Detection by Drones through Numerical Fluid Dynamics Simulations. Sensors, 20(6): 1770. https://doi.org/10.3390/s20061770

[43] www.MSAsafety.com.

[44] Haag, W., Wrenn, C. (2006). The PID Handbook-Theory and Applications of Direct-Reading Photoionization Detectors. Aufl.(RAE Systems Inc., San Jose, 2013).

[45] Daum, K.A., Watrous, M.G., Neptune, M.D., Michael, D.I., Hull, K.J., Evans, J.D. (2006). Data for First Responder Use of Photoionization Detectors for Vapor Chemical Constituents (No. INL/EXT-05-00165). Idaho National Laboratory (INL).

[46] Systems, R. (2008). SensorRAE Electrochemical Conditioning Station. RAE SYSTEMS. Available: https://www.raesystems.com/sites/default/files/content/r esources/Manual_SensorRAE_UserGuide_032-4006000_RevB.pdf

[47] Systems, R. Chemical-Warfare-Agent-MeasurementsBy-PID. Available: https://www.raesystems.com/sites/default/files/content/r esources/Technical-Note-159_Chemical-WarfareAgent-Measurements-By-PID_03-06.pdf

[48] ELCOD project contribution. (2019). Available: http://www.elcod.eu/wp/wpcontent/uploads/2019/07/2019_elcod_5_pmeier.pdf

[49] Wang, C., Yin, L., Zhang, L., Xiang, D., Gao, R. (2010). Metal oxide gas sensors: sensitivity and influencing factors. $\quad$ sensors, $10(3)$ : 2088-2106. https://doi.org/10.3390/s100302088

[50] Masson, N., Piedrahita, R., Hannigan, M. (2015). Approach for quantification of metal oxide type semiconductor gas sensors used for ambient air quality monitoring. Sensors and Actuators B: Chemical, 208: 339-345. https://doi.org/10.1016/j.snb.2014.11.032

[51] Rossi, M., Brunelli, D. (2015). Autonomous gas detection and mapping with unmanned aerial vehicles. IEEE Transactions on Instrumentation and Measurement, 65(4): 765-775. https://doi.org/10.1109/TIM.2015.2506319

[52] Korotcenkov, G., Cho, B.K. (2017). Metal oxide composites in conductometric gas sensors: Achievements and challenges. Sensors and Actuators B: Chemical, 244: 182-210. https://doi.org/10.1016/j.snb.2016.12.117

[53] Knoll, G.F. (2010). Radiation Detection and Measurement. John Wiley \& Sons.
[54] AEP-66 NATO handbook for sampling and identification of biological and chemical, and radiological agents (SIBCRA), NATO Standardization Office (NSO), 2015.

[55] Kummer, N., Augustyns, B., Van Rompaey, D., De Meulenaere, K. (2019). Forensic investigation of incidents involving chemical threat agent: Presentation of the operating procedure developed in Belgium for a field-exercise. Forensic Science International, 299: 180186. https://doi.org/10.1016/j.forsciint.2019.03.037

[56] NATO, ATP-45 Warning and reporting and hazard prediction of chemical, biological, radiological and nuclear incidents (operators manual), 2020.

[57] S. I. p. 1. D. NBC, Procedure Di Campionamento Di Matrici Potenzialmente Contaminate Da Agenti Biologici, Chimici E Radiologici, Rieti: COMFORDOT, 2021.

[58] Puton, J., Namieśnik, J. (2016). Ion mobility spectrometry: Current status and application for chemical warfare agents detection. TrAC Trends in Analytical Chemistry, $\quad 85$ : 10-20. https://doi.org/10.1016/j.trac.2016.06.002

[59] Hill, C.A., Thomas, C.L.P. (2003). A pulsed corona discharge switchable high resolution ion mobility spectrometer-mass spectrometer. Analyst, 128(1): 55-60. https://doi.org/10.1039/B207558J

[60] IAEA. (2012). IAEA Regulations for the Safe Transport of Radioactive Material, WIEN.

[61] SMITHS DETECTION LTD. Technical Information LCD3.3. Available: https://www.smithsdetection.com/products/lcd-3-3/

[62] Parker, B. Gas and Liquid Sample Analyzer Filters Sample Filters. Available: https://www.parker.com/Literature/Gas\%20Separation $\% 20$ and\%20Filtration\%20Division/PDF\%20Files/Balst on_Gas_and_Liquid_Sample_Analyzer_Filters.pdf.

[63] SKC INC. Available: https://www.skcinc.com/catalog/index.php?cPath=1000 00000_101000000_101000550.

[64] Ouvry. Chemical simulants for CBRNE. 2003-2020. Available: simulants-for-bcrne/.

[65] Bartelt-Hunt, S.L., Knappe, D.R., Barlaz, M.A. (2008). A review of chemical warfare agent simulants for the study of environmental behavior. Critical Reviews in Environmental Science and Technology, 38(2): 112-136. https://doi.org/10.1080/10643380701643650

[66] Pounds, P.E., Bersak, D.R., Dollar, A.M. (2011). Grasping from the air: Hovering capture and load stability. In 2011 IEEE International Conference on Robotics and Automation, pp. 2491-2498. https://doi.org/10.1109/ICRA.2011.5980314

[67] Marturano, F., Martellucci, L., Chierici, A., Malizia, A., Di Giovanni, D., D'errico, F., et al. (2021). Numerical fluid dynamics simulation for drones' chemical detection. In 2021 Drones, 5(3): https://doi.org/10.3390/drones5030069 